\title{
Investigating Gluino Production at the LHC
}

\author{
C. Brenner Mariotto and M. C. Rodriguez \\ Departamento de Física, Fundação Universidade Federal do Rio Grande \\ Caixa Postal 474, CEP 96201-900, Rio Grande, RS, Brazil
}

(Received on 14 April, 2008)

\begin{abstract}
Gluinos are expected to be one of the most massive sparticles (supersymmetric partners of usual particles) which constitute the Minimal Supersymmetric Standard Model (MSSM). The gluinos are the partners of the gluons and they are color octet fermions, due this fact they can not mix with the other particles. Therefore in several scenarios, given at SPS convention, they are the most massive particles and their nature is a Majorana fermion. Therefore their production is only feasible at a very energetic machine such as the Large Hadron Collider (LHC). Being the fermion partners of the gluons, their role and interactions are directly related with the properties of the supersymmetric QCD (sQCD). We review the mechanisms for producing gluinos at the LHC and investigate the total cross section and differential distributions, making an analysis of their uncertainties, such as the gluino and squark masses, as obtained in several scenarios, commenting on the possibilities of discriminating among them.
\end{abstract}

Keywords: Supersymmetric models; Supersymmetric partners of known particles

Although the Standard Model (SM) [1], based on the gauge symmetry $S U(3)_{c} \otimes S U(2)_{L} \otimes U(1)_{Y}$ describes the observed properties of charged leptons and quarks it is not the ultimate theory. However, the necessity to go beyond it, from the experimental point of view, comes at the moment only from neutrino data. If neutrinos are massive then new physics beyond the $\mathrm{SM}$ is needed.

Although the SM provides a correct description of virtually all known microphysical nongravitacional phenomena, there are a number of theoretical and phenomenological issues that the SM fails to address adequately [2]:

- Hierarchy problem;

- Electroweak symmetry breaking (EWSB);

- Gauge coupling unification.

The main sucess of supersymmetry (SUSY) is in solving the problems listed above.

SUSY has also made several correct predictions [2]:

- SUSY predicted in the early 1980s that the top quark would be heavy;

- SUSY GUT theories with a high fundamental scale accurately predicted the present experimental value of $\sin ^{2} \theta_{W}$ before it was mesured;

- SUSY requires a light Higgs boson to exist.

Together these success provide powerful indirect evidence that low energy SUSY is indeed part of correct description of nature.

Certainly the most popular extension of the SM is its supersymmetric counterpart called Minimal Supersymmetric Standard Model (MSSM) [3]. The main motivation to study this models, is that it provides a solution to the hierarchy problem by protecting the electroweak scale from large radiative corrections $[4,5]$. Hence the mass square of the lightest real scalar boson has an upper bound given by

$$
M_{h}^{2} \leq\left(M_{Z}^{2}+\varepsilon^{2}\right) \mathrm{GeV}^{2}
$$

where $M_{Z}^{2}$ is the $\mathrm{Z}$ mass. Therefore the $\mathrm{CP}$ even, light Higgs $h$, is expected lighter than $Z$ at tree level $(\varepsilon=0)$. However, radiative corrections rise it to $130 \mathrm{GeV}$ [6].

In the MSSM [3], the gauge group is $S U(3)_{C} \otimes S U(2)_{L} \otimes$ $U(1)_{Y}$. The particle content of this model consists in associate to every known quark and lepton a new scalar superpartner to form a chiral supermultiplet. Similarly, we group a gauge fermion (gaugino) with each of the gauge bosons of the standard model to form a vector multiplet. In the scalar sector, we need to introduce two Higgs scalars and also their supersymmetric partners known as Higgsinos. We also need to impose a new global $U(1)$ invariance usually called $R$-invariance, to get interactions that conserve both lepton and baryon number (invariance).

Other very popular extensions of SM are Left-Right symmetric theories [7], which attribute the observed parity asymmetry in the weak interactions to the spontaneous breakdown of Left-Right symmetry, i.e. generalized parity transformations. It is characterized by a number of interesting and important features [8]:

1. it incorporates Left-Right (LR) symmetry which leads naturally to the spontaneous breaking of parity and charge conjugation;

2. incorporates a see-saw mechanism for small neutrino masses.

On the technical side, the left-right symmetric model has a problem similar to that in the SM: the masses of the fundamental Higgs scalars diverge quadratically. As in the SM, the Supersymmetric Left-Right Model (SUSYLR) can be used to stabilize the scalar masses and cure this hierarchy problem.

Another, maybe more important raison d'etre for SUSYLR models is the fact that they lead naturally to R-parity conservation [9]. Namely, Left-Right models contain a $B-L$ gauge symmetry, which allows for this possibility [10]. All that is needed is that one uses a version of the theory that incorporates a see-saw mechanism [11] at the renormalizable level.

The supersymmetric extension of left-right models $[12,13]$ is based on the gauge group $S U(3)_{C} \otimes S U(2)_{L} \otimes S U(2)_{R} \otimes$ 
$U(1)_{B-L}$. On the literature there are two different SUSYLR models. They differ in their $S U(2)_{R}$ breaking fields: one uses $S U(2)_{R}$ triplets [12] (SUSYLRT) and the other $S U(2)_{R}$ doublets [13] (SUSYLRD). Since we are interested in studying only the strong sector, which is the same in both models, the results we are presenting here hold in both models.

As a result of a more detailed study, we have shown that the Feynman rules of the strong sector are the same in both MSSM and SUSYLR models [14]. The relevant Feynman rules for the gluino production are:

- Gluino-Gluino-Gluon: $-g_{s} f^{b a c}$;

- Quark-Quark-Gluon: $-l_{s} T_{r s}^{a} \gamma^{m}$ (usual QCD);

- Squark-Squark-Gluon: $-{ }^{-} g_{s} T_{r s}^{a}\left(k_{i}+k_{j}\right)^{m}$, where $k_{i, j}$ are the momentum of the incoming and outcoming squarks, respectively;

- Quark-Squark-Gluino: $-l \sqrt{2} g_{s}\left(L T_{r s}^{a}-R T_{r s}^{a}\right)$, where $L=$ $\frac{1}{2}\left(1-\gamma_{5}\right), R=\frac{1}{2}\left(1+\gamma_{5}\right)$.

The "Snowmass Points and Slopes" (SPS) [15] are a set of benchmark points and parameter lines in the MSSM parameter space corresponding to different scenarios in the search for Supersymmetry at present and future experiments. The aim of this convention is reconstructing the fundamental supersymmetric theory, and its breaking mechanism, from the data. The points SPS 1-6 are Minimal Supergravity (mSUGRA) model, SPS 7-8 are gauge-mediated symmetry breaking (GMSB) model, and SPS 9 are anomaly-mediated symmetry breaking (mAMSB) model ([15-17]). Each set of parameters leads to different gluino and squark masses, wich are the only relevant parameters in our study, and are shown in Tab.(I).

\begin{tabular}{|c|c|c|}
\hline \hline Scenario & $m_{\tilde{g}}(\mathrm{GeV})$ & $m_{\tilde{q}}(\mathrm{GeV})$ \\
\hline \hline SPS1a & 595.2 & 539.9 \\
SPS1b & 916.1 & 836.2 \\
SPS2 & 784.4 & 1533.6 \\
SPS3 & 914.3 & 818.3 \\
SPS4 & 721.0 & 732.2 \\
SPS5 & 710.3 & 643.9 \\
SPS6 & 708.5 & 641.3 \\
SPS7 & 926.0 & 861.3 \\
SPS8 & 820.5 & 1081.6 \\
SPS9 & 1275.2 & 1219.2 \\
\hline \hline
\end{tabular}

TABLE I: The values of the masses of gluinos and squarks in the SPS scenarios.

Gluino and squark production at hadron colliders occurs dominantly via strong interactions. Thus, their production rate may be expected to be considerably larger than for sparticles with just electroweak interactions whose production was widely studied in the literature $[18,19]$. Since the Feynman rules of the strong sector are the same in both MSSM and SUSYLR models, the diagrams that contribute to the gluino production are the same in both models.

In the present contribution we study the gluino production in $p p$ collisions at LHC energies. To make a consistent

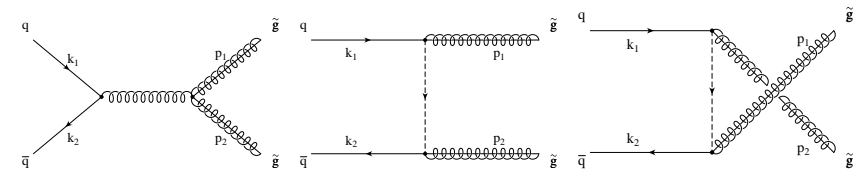

(a)

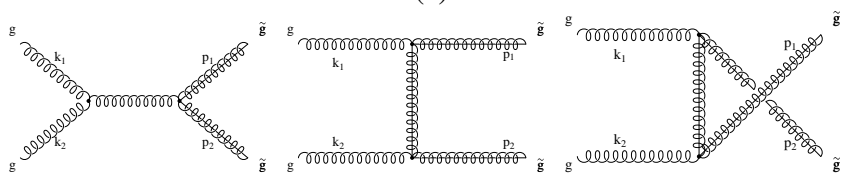

(b)

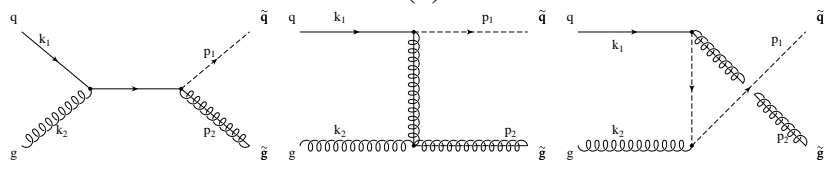

(c)

FIG. 1: Feynman diagrams for single $(a, b, c)$ and double $(a, b)$ gluino pair production.

comparison and for sake of simplicity, we restrict ourselves to leading-order (LO) accuracy, where the partonic crosssections for the production of squarks and gluinos in hadron collisions were calculated at the Born level already quite some time ago [20]. The corresponding NLO calculation has already been done for the MSSM case [21], and the impact of the higher order terms is mainly on the normalization of the cross section, which could be taken into account here by introducing a $\mathrm{K}$ factor in the results here obtained [21].

The LO QCD subprocesses for single gluino production are gluon-gluon and quark-antiquark anihilation $(g g \rightarrow \tilde{g} \tilde{g}$ and $q \bar{q} \rightarrow \tilde{g} \tilde{g})$, and the Compton process $q g \rightarrow \tilde{g} \tilde{q}$, as shown in Fig. 1. For double gluino production only the anihilation processes contribute, obviously. These two kinds of events could be separated, in principle, by analysing the different decay channels for gluinos and squarks [18, 19].

Incoming quarks (including incoming $b$ quarks) are assumed to be massless, such that we have $n_{f}=5$ light flavours. We only consider final state squarks corresponding to the light quark flavours. All squark masses are taken equal to $m_{\tilde{q}}{ }^{1}$. We do not consider in detail top squark production where these assumptions do not hold and which require a more dedicated treatment [22].

The invariant cross section for single gluino production can be written as [20]

$$
\begin{gathered}
E \frac{d \sigma}{d^{3} p}=\sum_{i j d} \int_{x_{\text {min }}}^{1} d x_{a} f_{i}^{(a)}\left(x_{a}, \mu\right) f_{j}^{(b)}\left(x_{b}, \mu\right) \\
\frac{x_{a} x_{b}}{x_{a}-x_{\perp}\left(\frac{\zeta+\cos \theta}{2 \sin \theta}\right)} \frac{d \hat{\sigma}}{d \hat{t}}(i j \rightarrow \tilde{g} d),
\end{gathered}
$$

where $f_{i, j}$ are the parton distributions of the incoming protons

\footnotetext{
${ }^{1} L$-squarks and $R$-squarks are therefore mass-degenerate and experimentally indistinguishable.
} 
and $\frac{d \hat{\sigma}}{d \hat{t}}$ is the LO partonic cross section [20] for the subprocesses involved. The identified gluino is produced at centerof-mass angle $\theta$ and transverse momentum $p_{T}$, and $x_{\perp}=\frac{2 p_{T}}{\sqrt{s}}$. The kinematic invariants of the partonic reactions $i j \rightarrow \tilde{g} \tilde{g}, \tilde{g} \tilde{q}$ are then

$$
\begin{aligned}
& \hat{s}=x_{a} x_{b} s, \\
& \hat{t}=m_{\tilde{g}}^{2}-x_{a} x_{\perp} s\left(\frac{\zeta-\cos \theta}{2 \sin \theta}\right), \\
& \hat{u}=m_{\tilde{g}}^{2}-x_{b} x_{\perp} s\left(\frac{\zeta+\cos \theta}{2 \sin \theta}\right) .
\end{aligned}
$$

Here

$$
\begin{aligned}
x_{b} & =\frac{2 v+x_{a} x_{\perp} s\left(\frac{\zeta-\cos \theta}{\sin \theta}\right)}{2 x_{a} s-x_{\perp} s\left(\frac{\zeta+\cos \theta}{\sin \theta}\right)}, \\
x_{\min } & =\frac{2 v+x_{\perp} s\left(\frac{\zeta+\cos \theta}{\sin \theta}\right)}{2 s-x_{\perp} s\left(\frac{\zeta-\cos \theta}{\sin \theta}\right)}, \\
\zeta & =\left(1+\frac{4 m_{\tilde{g}}^{2} \sin ^{2} \theta}{x_{\perp}^{2} s}\right)^{1 / 2}, \\
v & =m_{d}^{2}-m_{\tilde{g}}^{2},
\end{aligned}
$$

where $m_{\tilde{g}}$ and $m_{d}$ are the masses of the final-state partons produced. The center-of-mass angle $\theta$ and the differential cross section above can be easilly written in terms of the pseudorapidity variable $\eta=-\ln \tan (\theta / 2)$, which is one of the experimental observables. The total cross section for the gluino production can be obtained from above upon integration.

In Fig.2 we present the LO QCD total cross section for gluino production at the LHC as a function of the gluino masses. We use the CTEQ6L [23], parton densities, with two assumptions on the squark masses and choices of the hard scale. The results show a strong dependence on the masses of gluinos and squarks, and also a larger cross section in the degenerated mass case, which agrees with the results presented at [18].

The search for gluinos and squarks (as well as other searches for SUSY particles) and the possibility of detecting them will depend on their real masses. We use the SPS values from Table I and proceed to the calculation of differential distributions for producing gluinos in all presented scenarios. From now on we restrict ourselves to the production of two gluinos, picking only the anihilation processes as explained above. The calculation of producing a single gluino (including the Compton process) is done in a more detailed publication[14]. The results obtained will show the possibility of discriminating among the different SPS scenarios.

In Figs. 3 and 4 we present the transverse momentum and pseudorapidity distributions for double gluino production at LHC energies. The results show a similar behavior of the $p_{T}$ and $\eta$ dependencies in all scenarios, but a huge diference in the magnitude for different scenarios - SPS1a gives the bigger values, SPS9 the smallest one. Also, we find very close values for SPS1b, SPS3 (mSUGRA) and SPS7 (GMSB), which

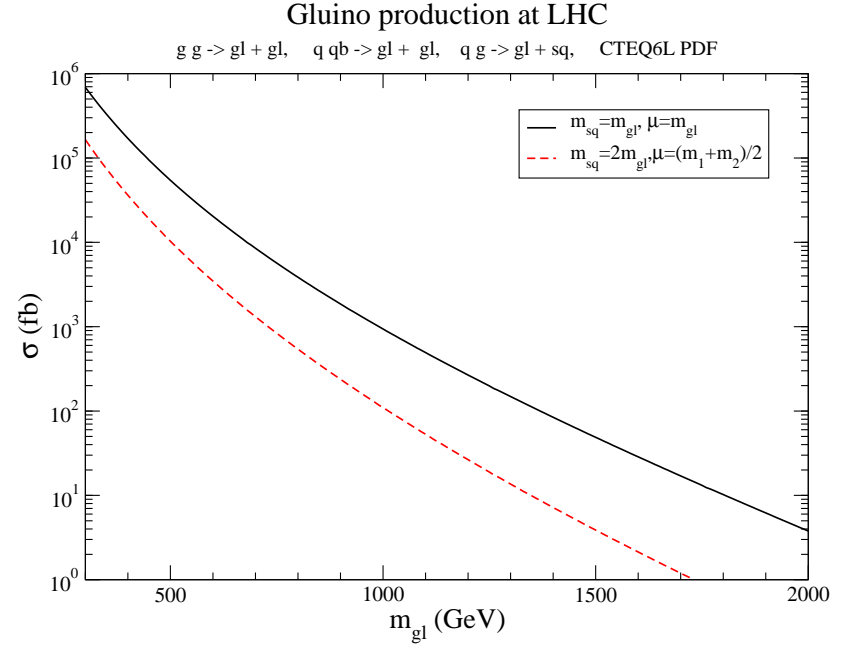

FIG. 2: The total LO cross section for gluino production at the LHC as a function of the gluino masses. Parton densities: CTEQ6L, with two assumptions on the squark masses and choices of the hard scale.

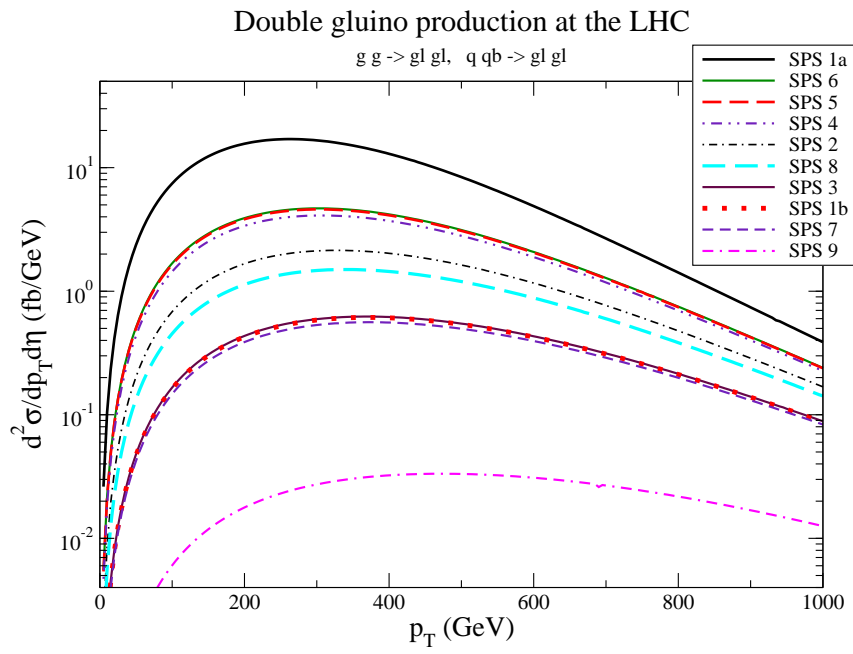

FIG. 3: The LO $p_{T}$ distributions for gluino production at the LHC for the different SPS points $[15,16]$. We use CTEQ6L parton densities, and $\mu^{2}=m_{\tilde{g}}^{2}+p_{T}^{2}$ as a hard scale.

makes difficult to discriminate between these mSUGRA and GMSB models. The same occurs for SPS5 and SPS6 (both mSUGRA).

To conclude, we have investigated gluino production at the LHC, which might discover supersymmetry over the next years. Gluinos are color octet fermions and play a major role to understanding $\mathrm{sQCD}$. Because of their large mass as predicted in several scenarios, up to now the LHC is the only possible machine where they could be found.

Regarding the strong sector, the Feynman rules are the same for both MSSM and SUSYLR models. Therefore, our results for gluino production are equal in both models. Besides, our results depend on the gluino and squark masses and no other SUSY parameters. Since the masses of gluinos come only from the soft terms, measuring their masses can test the soft 


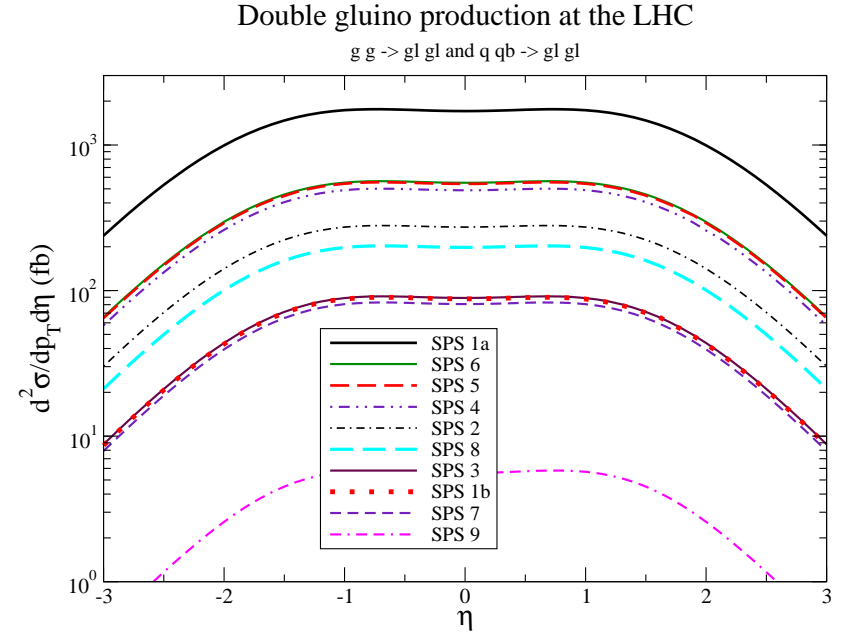

FIG. 4: The LO pseudorapidity distributions for gluino production at the LHC for the different SPS points $[15,16]$. We use CTEQ6L parton densities, and $\mu^{2}=m_{\tilde{g}}^{2}+p_{T}^{2}$ as a hard scale.
SUSY breaking approximations. We have considered all the SPS scenarios and showed the corresponding differences on the magnitude of the production cross sections. From this it is easy to distinguish mAMSB from the other scenarios. However, it is not so easy to distinguish mSUGRA from GMSB depending on the real values of masses of gluinos and squarks (if SPS1b and SPS7, provided the gluino and squark masses are almost similar in these two cases). For the other cases, such discrimination can be done.

\section{Acknowledgments}

This work was partially financed by the Brazilian funding agency CNPq, CBM under contract number 472850/2006-7, and MCR under contract number 309564/2006-9.
[1] S. L. Glashow, Nucl. Phys. 22, 579 (1961); S. Weinberg, Phys. Rev. Lett. 19, 1264 (1967); A. Salam in Elementary Particle Theory: Relativistic Groups and Analyticity, Nobel Symposium N8 (Alquivist and Wilksells, Stockolm, 1968); S. L. Glashow, J.Iliopoulos and L.Maini, Phys. Rev. D 2, 1285 (1970).

[2] D. J. H. Chung, L. L. Everett, G. L. Kane, S. F. King, J. D. Lykken, and L. T. Wang, Phys. Rept. 407, 1 (2005).

[3] H. E. Haber and G. L. Kane, Phys. Rep. 117, 75 (1985).

[4] K. Inoue, A. Komatsu, and S. Takeshita, Prog. Theor. Phys. 68 , 927 (1982)

[5] K. Inoue, A. Komatsu, and S. Takeshita, Prog. Theor. Phys. 70, 330 (1983).

[6] H. E. Haber, Eur. Phys. J. C 15, 817 (2000).

[7] J. C. Pati and A. Salam, Phys. Rev. D 10, 275 (1974); R. N. Mohapatra and J. C. Pati, ibid D 11, 566; 2558 (1975); G. Senjanović and R.N. Mohapatra, ibid D 12, 1502 (1975). For details see G. Senjanović, Nucl. Phys. B 153, 334 (1979).

[8] A. Melfo and G. Senjanović, Phys. Rev. D 68, 035013 (2003).

[9] C. S. Aulakh, A. Melfo, and Goran Senjanović , Phys. Rev. D 57, 4174 (1998).

[10] R. N. Mohapatra, Phys. Rev. D 34, 3457 (1986); A. Font, L. E Ibáñez, and F. Quevedo, Phys. Lett. B 228, 79 (1989); L. Ibáñez and G. Ross, Phys. Lett. B 260, 291 (1991); S. P. Martin, Phys. Rev. D 46, 2769 (1992).

[11] M. Gell-Mann, P. Ramond, and R. Slansky, in Supergravity, eds. P. van Niewenhuizen and D. Z. Freedman (North Holland 1979); T. Yanagida, in Proceedings of Workshop on Unified Theory and Baryon number in the Universe, eds. O. Sawada and A. Sugamoto (KEK 1979); R. N. Mohapatra and G. Senjanović, Phys. Rev. Lett. 44, 912 (1980).

[12] K. Huitu, J. Maalampi, and M. Raidal, Nucl. Phys. B 420, 449 (1994); C. S. Aulakh, A. Melfo, and G.Senjanovic, Phys. Rev. D 57, 4174 (1998); G. Barenboim and N. Rius, Phys. Rev. D 58, 065010 (1998); N. Setzer and S. Spinner, Phys. Rev. D 71, 115010 (2005).

[13] K. S. Babu. B. Dutta, and R. N. Mohapatra, Phys.Rev. D 65, 016005 (2002).

[14] C. B. Mariotto and M. C. Rodriguez, arXiv:0805.2094 [hep$\mathrm{ph}$.

[15] B. C. Allanach et al, Eur. Phys. J. C 25, 113 (2002).

[16] Nabil Ghodbane, Hans-Ulrich Martyn, hep-ph/0201233.

[17] http://spa.desy.de/spa/

[18] H. Baer and X. Tata, Weak Scale Supersymmetry, Cambridge University Press, United Kindom, (2006).

[19] M. Dress, R. M. Godbole, and P. Roy, Theory and Phenomenology of Sparticles, World Scientific Publishing Co. Pte. Ltd., Singapore, (2004).

[20] S. Dawson, E. Eichten, and C. Quigg, Phys. Rev. D 31, 1581 (1985).

[21] W. Beenakker, R. Höpker, M. Spira, and P. M. Zerwas, Nucl. Phys. B 492, 51 (1997).

[22] W. Beenakker, M. Krämer, T. Plehn, M. Spira, and P. M. Zerwas, Nucl. Phys. B 515, 3 (1998).

[23] J. Pumplin, D. R. Stump, J. Huston, H. L. Lai, P. Nadolsky, and W. K. Tung, JHEP 0207, 012 (2002). 